\title{
On Ignoring the Singularity in Numerical Quadrature
}

\author{
By R. K. Miller*
}

\begin{abstract}
This paper studies the convergence of numerical quadratures of singular integrands. The singularities are ignored in the sense that whenever a singularity occurs the integrand is redefined to be zero. Several convergence theorems are proved under the assumption that the integrand can be dominated near each singularity by a monotone, integrable function.
\end{abstract}

1. Introduction. Davis and Rabinowitz [1] recently studied the question of "ignoring the singularity" in numerical quadrature. That is, if $f(t)$ becomes singular at a point $\xi$ where $a \leqq \xi \leqq b$, then one defines $f(\xi)=0$ (or any other finite value) and then approximates the integral

$$
I=\int_{a}^{b} f(t) d t
$$

by a usual numerical quadrature rule. They show that, in general, one obtains only "lim inf convergence". However, if $\xi=a$ (or some other "rational" point of $[a, b]$ ), then compound quadrature rules do approximate $I$ when $f$ is monotone near $\xi$. Certain of the positive results in [1] were generalized by Rabinowitz [2]. Gautschi [3] has applied a result in [2] to two other quadratures of interpolatory type.

The purpose of this paper is to generalize some of the convergence theorems in [1] and [2]. We shall replace the assumption of monotonicity of $f(t)$ near $t=\xi$ by the more general condition that $f(t)$ can be dominated by a monotone, integrable function. We shall also establish some theorems on error bounds and convergence rates.

2. General Quadratures. Let $M$ be the set

$$
M=\left\{f \in C(0, T] \cap L^{1}(0, T): f \text { is nonnegative and nonincreasing on } 0<t \leqq T\right\} .
$$

Define $M_{d}$ to be the set of all functions $f \in C(0, T]$ such that $f$ can be majorized by a function in $M$,

$$
M_{d}=\{f \in C(0, T]: \exists F \in M \text { with }|f(t)| \leqq F(t) \text { on } 0<t \leqq T\} .
$$

(For example $f(t)=t^{-\alpha} \sin \left(t^{-1}\right)$ is in $M_{d}$ if $0<\alpha<1$, and is not in $M_{d}$ if $\alpha \geqq 1$.) For any function $f \in M_{d}$ assign the arbitrary value $f(t)=0$ when $t=0$.

Given any numerical quadrature rule

$$
Q(f)=\sum_{j=0}^{n} W(j) f(T(j)), \quad f \in C[0, T],
$$

Received December 22, 1969, revised March 11, 1970.

AMS 1969 subject classifications. Primary 6555.

Key words and phrases. Quadrature, singular quadrature, singular integrals, ignoring the singularity, error bounds, weak singularity.

* This research was supported in part by the National Aeronautics Space Administration under Grant No. NGL-40-002-015, and in part by the Air Force Office of Scientific Research under Grant No. AF-AFOSR-67-0693A. 
where $0 \leqq T(0)<T(1)<\cdots<T(n) \leqq T$.

Let $Q(f, S)$ be the modified quadrature rule obtained from $Q$ by redefining $f(0)=0$,

$$
Q(f, S)=\sum_{i=0}^{n} W(j) f(T(j)), \quad f(0)=0 .
$$

For example, if $Q(f)=\frac{1}{3}\left\{f_{0} / 2+f_{1}+f_{2}+f_{3} / 2\right\}$ is the compound trapezoid rule $3 \times T$, then $Q(f, S)=\frac{1}{3}\left\{f_{1}+f_{2}+f_{3} / 2\right\}$. Therefore, the modified rule $Q(f, S)$ ignores possible singularities in $f(t)$ at $t=0$. This modified rule is well defined for all functions $f \in M_{d}$. For open rules $Q(f, S) \equiv Q(f)$, while for closed rules $Q(f, S) \neq Q(f)$, in general, for all functions $f \in C[0, T]$.

With these preliminaries, we are now ready to generalize the lemma in [2, p. 194]. First, consider rules which are open at $t=0$.

LEMMA 1. Consider a sequence of rules

$$
Q_{n}(f)=\sum_{i=0}^{m_{n}} W_{n}(j) f\left(T_{n}(j)\right),
$$

where $0<T_{n}(0)<T_{n}(1)<\cdots<T_{n}\left(m_{n}\right) \leqq T$ and $W_{n}(j)>0$ for all $j$. Define $T_{n}(-1)=0$. Suppose there exist positive constants $C$ and $A$ which are independent of $n=1,2,3, \cdots$ and of $j=0(1) m_{n}$ such that $A \leqq T$ and such that if $T_{n}(j)<A$ then

$$
W_{n}(j) \leqq C\left\{T_{n}(j)-T_{n}(j-1)\right\} .
$$

$\left(j=0(1) m_{n}\right.$ means $j=0,1,2, \cdots, m_{n}$.

Suppose for each function $g \in C[0, T]$ one has

$$
\lim Q_{n}(g)=\int_{0}^{T} g(t) d t, \quad n \rightarrow \infty .
$$

Then, for any function $f \in M_{d}$,

$$
\lim Q_{n}(f, S)=\int_{0}^{T} f(t) d t, \quad n \rightarrow \infty .
$$

In particular, if $0<B<A$, if one defines

$$
\begin{aligned}
f_{B}(t) & =f(t) \quad \text { on } B \leqq t \leqq T, \\
& =f(B) \quad \text { on } 0 \leqq t \leqq B,
\end{aligned}
$$

and if

$$
\delta(t)=\sup \left\{\left|f(s)-f_{B}(s)\right|: t \leqq s \leqq T\right\},
$$

then the error

$$
E_{s}\left(f, Q_{n}\right)=\int_{0}^{T} f(t) d t-Q_{n}(f, S)
$$

satisfies the estimate

$$
\left|E_{s}\left(f, Q_{n}\right)\right| \leqq\left|E\left(f_{B}, Q_{n}\right)\right|+\left|\int_{0}^{B}\{f(t)-f(B)\} d t\right|+C \int_{0}^{B} \delta(t) d t
$$


Proof. Write $E=E_{a}\left(f, Q_{n}\right)$ in the form

$$
\begin{aligned}
E= & \int_{0}^{T}\left\{f(t)-f_{B}(t)\right\} d t+E\left(f_{B}, Q_{n}\right) \\
& +\sum_{i=0}^{m_{n}} W_{n}(j)\left\{f_{B}\left(T_{n}(j)\right)-f\left(T_{n}(j)\right)\right\} \\
= & \int_{0}^{B}\{f(t)-f(B)\} d t+E\left(f_{B}, Q_{n}\right)+\epsilon_{n} .
\end{aligned}
$$

Then, for any $n$,

$$
\begin{aligned}
\left|\epsilon_{n}\right| & \leqq \sum_{j=0}^{m_{n}} W_{n}(j)\left|f\left(T_{n}(j)\right)-f_{B}\left(T_{n}(j)\right)\right| \\
& \leqq \sum_{i=0}^{m_{n}} W_{n}(j) \delta\left(T_{n}(j)\right)=Q_{n}(\delta, S) .
\end{aligned}
$$

Since $f \in M_{d}$, there exists a majorizing function $F \in M$. Then, for $s$ in the range $0<t \leqq s<B$, one has

$$
\left|f(s)-f_{B}(s)\right| \leqq F(s)+F(B) \leqq 2 F(t) .
$$

Therefore, $\delta(t) \leqq 2 F(t)$ on $0<t \leqq B, \delta(t)=0$ on $B \leqq t \leqq 1$, and hence $\delta \in L^{1}(0,1)$. Note also that $\delta(t)$ is continuous, nonnegative and nonincreasing on the interval $0<t \leqq 1$. This together with (2.1) implies that

$$
\begin{aligned}
Q_{n}(\delta, S) & =\sum_{T_{n}(j)<B} W_{n}(j) \delta\left(T_{n}(j)\right) \leqq C \sum\left\{T_{n}(j)-T_{n}(j-1)\right\} \delta\left(T_{n}(j)\right) \\
& \leqq C \sum \int_{T_{n}(i-1)}^{T_{n}(i)} \delta(t) d t=C \int_{0}^{B} \delta(t) d t
\end{aligned}
$$

This completes the proof of (2.4).

Line (2.3) follows immediately from (2.4) and the estimate $\delta(t) \leqq 2 F(t)$. Indeed, by first choosing $B$ small and then choosing $n$ large, one can make the right-hand side of (2.4) as small as desired. Q.E.D.

Although Rabinowitz only studies rules which are open at the singularity, almost the same result is true for quadratures which are closed there.

LEMMA 2. Suppose a sequence of quadrature rules $Q_{n}$ satisfies the two conditions

$$
0=T_{n}(0)<T_{n}(1)<\cdots<T_{n}\left(m_{n}\right) \leqq T, \quad W_{n}(j)>0 .
$$

Suppose there exist positive constants $C$ and $A$ such that if $T_{n}(j)<A$ and if $j=1(1) m_{n}$ then (2.1) is true uniformly in $n$. If (2.2) is also true, then (2.3) follows. In particular, if $f_{B} \in C[0, T]$ and $\delta \in M_{d}$ are the functions defined in Lemma 1 and if $0<B<A$, then

$$
\begin{aligned}
\left|E_{s}\left(f, Q_{n}\right)\right| \leqq & \left|E\left(f_{B}, Q_{n}\right)\right|+\left|\int_{0}^{B}\{f(t)-f(B)\} d t\right| \\
& +C \int_{0}^{B} \delta(t) d t+W_{n}(0)|f(B)| .
\end{aligned}
$$


Proof. The proof is the same as that of Lemma 1 except for the estimates of $\epsilon_{n}$. In this case

$$
\epsilon_{n}=\sum_{i=0}^{m_{n}} W_{n}(j)\left\{f\left(T_{n}(j)\right)-f_{B}\left(T_{n}(j)\right)\right\}
$$

and

$$
\begin{aligned}
\left|\epsilon_{n}\right| & \leqq Q_{n}(\delta, S)+W_{n}(0)|f(B)| \\
& \leqq C \int_{0}^{B} \delta(t) d t+W_{n}(0)|f(B)|,
\end{aligned}
$$

where we define $\delta(0)=0$. The proof will be complete if we show that $W_{n}(0) \rightarrow 0$ as $n \rightarrow \infty$. Let $d>0$ be given and define $f_{d}(t)=1-t / d$ if $0 \leqq t \leqq d$ and $f_{d}(t)=0$ if $t \geqq d$. By (2.2) it follows that, as $n \rightarrow \infty$,

$$
0 \leqq W_{n}(0) \leqq Q\left(f_{d}, S\right) \rightarrow d / 2 .
$$

Therefore, $\lim \sup _{n} W_{n}(0) \leqq d / 2$ for each $d>0$. Q.E.D.

Combining the two results we have proved:

THEOREM 1. Consider a sequence of numerical quadrature rules $Q_{n}$ where $0 \leqq T_{n}(0)<T_{n}(1)<\cdots<T_{n}\left(m_{n}\right) \leqq T$ and $W_{n}(j)>0$. Suppose (2.1) is true as in Lemma 1 (when $T_{n}(0)>0$ ) or Lemma $2\left(T_{n}(0)=0\right)$. If $(2.2)$ is also true, then for any $f \in M_{d}$,

$$
Q_{n}(f, S) \rightarrow \int_{0}^{T} f(t) d t \quad \text { as } n \rightarrow \infty
$$

Indeed, if $0<B<A$ and if $f_{B} \in C[0, T]$ and $\delta \in M_{d}$ are the functions defined in Lemma 1, then

$$
\begin{aligned}
\left|E_{s}\left(f, Q_{n}\right)\right| \leqq & \left|E\left(f_{B}, Q_{n}\right)\right|+\left|\int_{0}^{B}\{f(t)-f(B)\} d t\right| \\
& +C \int_{0}^{B} \delta(t) d t+\left\{1-\operatorname{sgn} T_{n}(0)\right\} W_{n}(0)|f(B)| .
\end{aligned}
$$

Rabinowitz [2, Corollary, p. 196] also studied integrals of the form $\int_{0}^{t} r(t) f(t) d t$ where $r(t)$ is a positive weight function. Since

$$
\sum W_{n}(j) f\left(T_{n}(j)\right)=\sum\left\{W_{n}(j) / r\left(T_{n}(j)\right)\right\} r\left(T_{n}(j)\right) f\left(T_{n}(j)\right),
$$

this reduces to the previous case with new weights $\left\{W_{n}(j) / r\left(T_{n}(j)\right)\right\}$ and a new integrand $r(t) f(t)$ on $0<t \leqq T$. If one assumes that $r(t) f(t) \in M_{d}$, then this corollary can be generalized in the obvious way.

3. Compound Rules. Consider a quadrature rule $R$ defined on the interval $0 \leqq t \leqq 1$ :

$$
R(t)=\sum_{i=0}^{J} W_{i} f\left(t_{i}\right)
$$

where $J \geqq 0$ and

$$
0 \leqq t_{0}<t_{1}<\cdots<t_{J} \leqq 1, \quad W_{j}>0, \quad \sum_{i=0}^{J} W_{i}=1 .
$$


(If $t_{0}>0$, then define $t_{-1}=0$.) For any integer $n \geqq 1$ and any interval $0 \leqq t \leqq T$, one can then define a compound rule

$(n \times R) \quad R_{n}(f)=\sum_{k=0}^{n-1}\left\{\sum_{i=0}^{J} H W_{i} f\left(t_{i} H+H k\right)\right\}$,

where $H=T / n$. Let $C>0$ be any constant satisfying

$$
W_{i} \leqq\left(t_{i}-t_{i-1}\right) C \quad(j=1(1) J)
$$

and either

$$
W_{0} \leqq\left(t_{0}+1-t_{J}\right) C \quad\left(\text { if } t_{0}>0 \text { or } t_{J}<1\right)
$$

or

$$
\left.\left(W_{0}+W_{J}\right) \leqq\left(t_{J}-t_{J-1}\right) C \quad \text { (if } t_{0}=0 \text { and } t_{J}=1\right) .
$$

THEOREM 2. If $(R)$ satisfies (3.1), then for any $f \in M_{d}$

$$
\lim _{n \rightarrow \infty} R_{n}(f, S)=\int_{0}^{T} f(t) d t
$$

Proof. The definition (3.2-3.3a, b) of $C$ implies that (2.1) is true with $A=T$. Since $R$ integrates constants and $n \rightarrow \infty$, then (2.2) is also trivial. Therefore, Theorem 2 is a corollary of Theorem 1. Q.E.D.

The error estimate (2.6) is rather pessimistic for compound rules. Therefore, we shall derive another estimate. which is more suitable for many purposes. Let $K>0$ be the smallest constant which satisfies

$$
W_{i} \leqq\left(t_{i}-t_{i-1}\right) K
$$

for $j=1(1) J$ if $t_{0}=0$ or for $j=0(1) J$ if $t_{0}>0$.

THEOREM 3. Suppose (3.1) and (3.4) are satisfied. Let $H=T / n$. For any function $f \in M_{d}$, define

$$
\begin{aligned}
f_{H}(t) & =f(H) \quad \text { if } 0 \leqq t \leqq H, \\
& =f(t) \quad \text { if } H \leqq t \leqq T .
\end{aligned}
$$

If $F \in M$ is any majorizing function for $f$, then

$$
\left|E_{8}\left(f, R_{n}\right)\right| \leqq\left|E\left(f_{H}, R_{n}\right)\right|+\int_{0}^{H} F(t) d t+K \int_{0}^{t J^{H}} F(t) d t
$$

or

$$
\left|E_{s}\left(f, R_{n}\right)\right| \leqq\left|E\left(f_{H}, R_{n}\right)\right|+(1+K) \int_{0}^{H} F(t) d t .
$$

Proof. Since $(R)$ integrates constants, then the error may be written in the form $E_{\text {。 }}\left(f, R_{n}\right)=E\left(f_{H}, R_{n}\right)+E_{0}$, where

$$
E_{0}=\int_{0}^{H} f(t) d t-\sum_{i=0}^{J} H W_{i} f\left(t_{i} H\right) .
$$


Since $|f(t)| \leqq F(t)$ on $0<t \leqq T$, then

$$
\left|\int_{0}^{H} f(t) d t\right| \leqq \int_{0}^{H} F(t) d t
$$

Let $\alpha=0$ if $t_{0}>0$ and $\alpha=1$ if $t_{0}=0$. Then $f(0)=0$ and (3.4) imply

$$
\begin{aligned}
\left|\sum_{i=0}^{J} H W_{i} f\left(t_{i} H\right)\right| & \leqq \sum_{\alpha}^{J} H W_{i} F\left(t_{i} H\right) \\
& \leqq K \sum_{\alpha}^{J} H\left(t_{i}-t_{i-1}\right) F\left(t_{i} H\right) \\
& \leqq \sum_{\alpha}^{J} \int_{H t_{i-1}}^{H t_{i}} F(t) d t=K \int_{0}^{t_{J} H} F(t) d t .
\end{aligned}
$$

This proves (3.5) and the theorem. Q.E.D.

If one knows that $f \in C^{1}(0, T]$, then the term $E\left(f_{H}, R_{n}\right)$ may be estimated using Peano's theorem. That is

$$
E\left(f_{H}, R_{n}\right)=\int_{0}^{1} P_{n}(s) f_{H}^{\prime}(s) d t
$$

where $P_{n}$ is the appropriate Peano kernel. Since $P_{n}(s+H)=P_{n}(s)$ on $0 \leqq s \leqq T-H$, then $\left|P_{n}(s)\right|$ need be estimated only on the interval $0<s<H$. Therefore the following result is an immediate corollary of Theorem 3.

COROLlaRY 1. Assume the hypotheses of Theorem 3. If $f \in C^{1}(0, T]$ then

$$
\left|E_{s}\left(f, R_{n}\right)\right| \leqq\left\|P_{n}\right\| \int_{H}^{T}\left|f^{\prime}(t)\right| d t+\int_{0}^{H} F(t) d t+K \int_{0}^{t_{J} H} F(t) d t
$$

where $\left\|P_{n}\right\|=\sup \left\{\left|P_{n}(s)\right|: 0<s<H\right\}$.

Corollary 1 is useful in estimating convergence rates in certain cases. We shall say that a function $f \in C^{1}(0, T]$ is weakly singular at $t=0$ if the function

$$
\alpha(t, f)=|f(T)|+\int_{t}^{T}\left|f^{\prime}(s)\right| d s
$$

is in $L^{1}(0, T)$. This definition was introduced and will be more completely investigated in some joint work of this author and A. Feldstein. Notice that $\alpha^{\prime}(t, f)=-\left|f^{\prime}(t)\right| \leqq 0$ if $t>0$. Thus $\alpha$ is nonnegative, monotone and $C(0, T]$, in particular $\alpha \in M$.

COROLlaRY 2. Suppose the hypotheses of Theorem 3 are true. If $f$ is weakly singular $($ at $t=0)$ then

$$
E_{s}\left(f, R_{n}\right)=O\left(\int_{0}^{H} \alpha(t, f) d t\right) \text { as } H \rightarrow 0 .
$$

Proof. It follows immediately from the definition of weakly singular functions that $f \in M_{d}$ and $\alpha(t, f) \in M$ is a majorizing function. Thus (3.7) implies

$$
\begin{aligned}
\left|E_{s}\left(f, R_{n}\right)\right| & \leqq\left\|P_{n}\right\| \int_{H}^{T}\left|f^{\prime}(t)\right| d t+\int_{0}^{H} \alpha(t, f) d t+K \int_{0}^{H} \alpha(t, f) d t \\
& \leqq\left\|P_{n}\right\| \alpha(H, f)+(1+K) \int_{0}^{H} \alpha(t, f) d t .
\end{aligned}
$$


The kernel $P_{n}(s)$ may easily be explicitly calculated using its definition (cf. Sard $[4$, p. 14]). Then

$$
P_{n}(s)=-s+\sum_{i=0}^{k} H W_{i}
$$

if $H t_{k}<s<H t_{k+1}$ and $k=0(1) J$. If $t_{0}>0$ or if $t_{J}<1$, a similar formula obtains in the end intervals. For $H t_{k}<s<H t_{k+1}$ and $k=0(1) J$, it follows from (3.1) that

$$
\left|P_{n}(s)\right| \leqq|s|+\sum_{j=0}^{k} H\left|W_{i}\right| \leqq H\left(1+\sum_{i=0}^{J} W_{i}\right)=2 H .
$$

Since $P_{n}(s+H)=P_{n}(s)$, then $\left\|P_{n}\right\| \leqq 2 H$. Using the estimate $\left\|P_{n}\right\| \leqq 2 H$ and the monotonicity of $\alpha$, one has

$$
\left\|P_{n}\right\| \alpha(H, f) \leqq 2 H \alpha(H, f) \leqq 2 \int_{0}^{H} \alpha(t, f) d t .
$$

Therefore, for any $n(H=T / n)$, one has

$$
\left|E_{s}\left(f, R_{n}\right)\right| \leqq(3+K) \int_{0}^{H} \alpha(t, f) d t . \quad \text { Q.E.D. }
$$

For example, if $f(t)=t^{-p}(0<p<1)$, then (3.8) predicts that $E_{0}\left(f, R_{n}\right)$ is at least of order $h^{1-p}$. If $f(t)=t^{-p} \sin \left(t^{-q}\right)$ where $0<p, q<1$ and $p+q<1$, then $E_{s}\left(f, R_{n}\right)$ is at least of order $h^{1-p-q}$. If $f(t)=t^{-p} \sin \left(t^{-q}\right)$ where $0<p<1, q>0$ and $p+q \geqq 1$, then our theory predicts convergence but gives no order estimate.

4. Singularities Between Zero and T. It is also possible to obtain convergence theorems for an integrable function $f$ which has a singularity at some point $\xi(0<\xi<T)$ and is otherwise continuous in the interval $0 \leqq t \leqq T$. For such functions, the two integrals

$$
\int_{0}^{\xi} f(t) d t \text { and } \int_{\xi}^{T} f(t) d t
$$

may be treated separately. The transformation $\tau=T-t$,

$$
\int_{0}^{\xi} f(t) d t=\int_{T-\xi}^{T} f(T-\tau) d \tau
$$

shows that it is sufficient to treat only integrals of the second type. Similar reductions can easily be made for integrable functions $f$ which have a finite number of singularities $\xi_{1}, \xi_{2}, \cdots, \xi_{n}$ in the interval $0 \leqq t \leqq T$.

Fix $\xi$ in the range $0<\xi<T$. Let $M(\xi)$ be the set defined by

$$
M(\xi)=\left\{f \in C(\xi, 1] \cap L^{1}(\xi, 1): f(t) \equiv 0 \text { on } 0 \leqq t \leqq \xi\right. \text { and }
$$

$f(t)$ is nonnegative and nonincreasing on $\xi<t \leqq T\}$.

Define $M_{d}(\xi)$ to be the set of all functions $f$ such that $f(t) \equiv 0$ on $0 \leqq t \leqq \xi$, $f \in C(\xi, 1]$ and $f$ can be majorized by a function $F \in M(\xi)$.

Given any quadrature rule $Q(f)$ defined for $f \in C[0, T]$ let $Q(f, \xi)$ be the modified 
rule obtained from $Q$ by setting $f(t)=0$ on $0 \leqq t \leqq \xi$ :

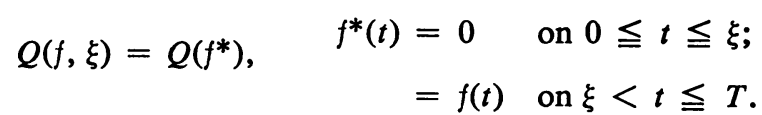

Let

$$
E_{\xi}(f, Q)=\int_{\xi}^{1} f(t) d t-Q(f, \xi)
$$

denote the error obtained by applying $Q(f, \xi)$.

The modified rule $Q(f, \xi)$ has the effect of first redefining $f(t) \equiv 0$ in $0 \leqq t<\xi$ and then applying $Q$ to the redefined function. For example, if $Q=3 \times T$ is the compounded trapezoid rule $Q(f)=\frac{1}{3}\left(f_{0} / 2+f_{1}+f_{2}+f_{3} / 2\right)$, then $Q(f, \xi)$ for $\xi=\frac{1}{2}$ has the form $Q(f, \xi)=\frac{1}{3}\left(f_{2}+f_{3} / 2\right)$. If $Q(f)$ is defined for all functions $f \in C[0, T]$, then clearly $Q(f, \xi)$ is well-defined for all $f \in M_{d}(\xi)$.

LEMMA 3. Suppose the rule $(R)$ satisfies (3.1). Let $R_{n}=n \times R$ be the corresponding compound rule. Suppose $f \in M_{d}(\xi)$ and $F \in M(\xi)$ is a majorizing function for $f$. For any $B$ such that $\xi<\xi+B<T$ define

$$
\begin{aligned}
f_{B}(t) & =f(t) & & \text { on } \xi+B \leqq t \leqq T, \\
& =f(\xi+B) & & \text { on } 0 \leqq t \leqq \xi+B,
\end{aligned}
$$

and define

$$
\delta_{B}(t)=\sup \left\{\left|f(s)-f_{B}(s)\right|: t<s \leqq T\right\} \quad(\xi<t \leqq T) .
$$

Then the error $E_{\xi}\left(f, R_{n}\right)$ satisfies the estimate

$$
\left|E_{\xi}\left(f, R_{n}\right)\right| \leqq\left|E_{\xi}\left(f_{B}, R_{n}\right)\right|+\left|\int_{0}^{B}\{f(t+\xi)-f(B+\xi)\} d t\right|+R_{n}\left(\delta_{B}, \xi\right) .
$$

Proof. By definition, $E=E_{\xi}\left(f, R_{n}\right)$ may be written in the form

$$
\begin{aligned}
E & =\int_{\xi}^{T}\left\{f(t)-f_{B}(t)\right\} d t+E_{\xi}\left(f_{B}, R_{n}\right)+R_{n}\left(f_{B}-f, \xi\right) \\
& =\int_{0}^{B}\{f(t+\xi)-f(B+\xi)\} d t+E_{\xi}\left(f_{B}, R_{n}\right)+R_{n}\left(f_{B}-f, \xi\right) .
\end{aligned}
$$

Write $R_{n}(g)$ in the form $R_{n}(g)=\sum_{j=0}^{n J} W_{n}(j) g\left(T_{n}(j)\right)$. Let $\xi(n)$ denote the smallest integer such that

$$
T_{n}(\xi(n))>\xi .
$$

Recalling that $\left|f(t)-f_{B}(t)\right| \leqq 2 F(t)$ on $\xi<t \leqq T$, it follows that

$$
\begin{aligned}
\left|R_{n}\left(f_{B}-f, \xi\right)\right| & =\left|\sum_{j=\xi(n)}^{n J} W_{n}(j)\left\{f_{B}\left(T_{n}(j)\right)-f\left(T_{n}(j)\right)\right\}\right| \\
& \leqq \sum_{i=\xi(n)}^{n J} W_{n}(j) \delta_{B}\left(T_{n}(j)\right)=R_{n}\left(\delta_{B}, \xi\right) \text {. Q.E.D. }
\end{aligned}
$$

LEMMA 4. Under the hypotheses of Lemma 3,

$$
\lim E_{\xi}\left(f_{B}, R_{n}\right)=0 \text { as } n \rightarrow \infty .
$$


Proof. For any $n$, let $k(n)$ and $j(n)$ be the unique integers such that

$$
(T / n)\left(k(n)+t_{i(n)}\right) \leqq \xi<(T / n)\left(k(n)+t_{i(n)+1}\right) .
$$

Since $R$ integrates constants one can write

$$
\begin{aligned}
E_{\xi}\left(f_{B}, R_{n}\right) & =E\left(f_{B}, R_{n}\right)+\sum_{i=0}^{i(n)}(T / n) W_{i} f_{B}\left(\left\{t_{i}+k(n)\right\}(T / n)\right) \\
& =E\left(f_{B}, R_{n}\right)+T f(\xi+B)\left(\sum_{i=0}^{i(n)} W_{i}\right) / n .
\end{aligned}
$$

But $f_{B} \in C[0, T]$ and $\left|\sum_{\substack{i=0 \\ i(n)}} W_{i}\right| \leqq 1$ by (3.1). The lemma follows. Q.E.D.

Lemma 5. Assume the hypotheses of Lemma 3. Then:

a. $\lim \inf _{n \rightarrow \infty}\left|R_{n}(f, \xi)-\int_{\xi}^{T} f(t) d t\right|=0$. then

b. If $R_{n}(F, \xi) \rightarrow \int_{\xi}^{T} F(t) d t$ as $n \rightarrow \infty$ or if for some $B>0, R_{n}\left(\delta_{B}, \xi\right) \rightarrow \int_{\xi}^{T} \delta_{B}(t) d t$,

$$
\lim _{n \rightarrow \infty} R_{n}(f, \xi)=\int_{\xi}^{T} f(t) d t
$$

Proof. Results in [1, Theorem 7] imply that for any $g \in M(\xi)$ one has $\lim \inf R_{n}(g, \xi)=\int_{\xi}^{T} g(t) d t$. Since $\delta_{B} \in M(\xi)$, this fact together with (4.1) and (4.3) imply that

$$
E_{0}=\liminf _{n \rightarrow \infty}\left|E_{\xi}\left(f, R_{n}\right)\right| \leqq 0+\left|\int_{0}^{B}\{f(t+\xi)-f(B+\xi)\} d t\right|+\int_{\xi}^{T} \delta_{B}(t) d t .
$$

But $\delta_{B}(t)=0$ if $t \geqq \xi+B$ and $\delta_{B}(t) \leqq 2 F(t)$ on $\xi<t<\xi+B$. Therefore,

$$
E_{0} \leqq\left|\int_{0}^{B}\{f(t+B)-f(B+\xi)\} d t\right|+2 \int_{0}^{B} F(t+\xi) d t .
$$

Letting $B \rightarrow 0^{+}$, we see that $E_{0}=0$. This proves part a.

Let $\xi(n)$ be the integer defined in (4.2). That is $T_{n}(\xi(n))$ is the smallest abscissa in the rule $R_{n}$ which is bigger than $\xi$. If $g \in M(\xi)$, then according to [1, Theorem 2],

$$
\limsup _{n \rightarrow \infty} R_{n}(g, \xi)=\int_{\xi}^{T} g(t) d t
$$

if and only if $g(\xi(n)) / n \rightarrow 0$ as $n \rightarrow \infty$. Recall that $\delta_{B}(t) \leqq 2 F(t)$ on $\xi<t<\xi+B$. Also if $0<b<B$, then for all $t$ in the interval $(\xi, \xi+b)$

$$
\begin{aligned}
\delta_{b}(t) & =\sup \{|f(s)-f(b+\xi)|: t<s<\xi+b\} \\
& \leqq \sup \{|f(s)-f(B+\xi)|: t<s<\xi+b\}+|f(B+\xi)-f(b+\xi)| \\
& \leqq \delta_{B}(t)+|f(B+\xi)-f(b+\xi)| .
\end{aligned}
$$

Therefore, if $F(\xi(n)) / n \rightarrow 0$ as $n \rightarrow \infty$, then $\delta_{B}(\xi(n)) / n \rightarrow 0$ for all small $B>0$. Also if for some fixed $B>0$ one knows that $\delta_{B}(\xi(n)) / n \rightarrow 0$ as $n \rightarrow \infty$, then for all $b$ in the range $0<b<B, \delta_{b}(\xi(n)) / n \rightarrow 0$.

In particular, the hypotheses of part $b$ always imply that

$$
\underset{n \rightarrow \infty}{\lim \sup } R_{n}\left(\delta_{B}, \xi\right)=\int_{\xi}^{T} \delta_{B}(t) d t=\int_{\xi}^{\xi+B} \delta_{B}(t) d t
$$


for all sufficiently small values of $B>0$.

From (4.1) and (4.3), we see that

$$
\begin{aligned}
E_{1} & =\underset{n \rightarrow \infty}{\lim \sup }\left|E_{\xi}\left(f, R_{n}\right)\right| \\
& \leqq 0+\left|\int_{0}^{B}\{f(t+\xi)-f(B+\xi)\} d t\right|+\underset{n \rightarrow \infty}{\lim \sup } R_{n}\left(\delta_{B}, \xi\right) .
\end{aligned}
$$

Hence, for $B$ sufficiently small,

$$
\begin{aligned}
E_{1} & \leqq\left|\int_{0}^{B}\{f(t+\xi)-f(B+\xi)\} d t\right|+\int_{\xi}^{\xi+B} \delta_{B}(t) d t \\
& \left.\leqq \mid \int_{0}^{B} f(t+\xi)-f(B+\xi)\right\} d t \mid+2 \int_{0}^{B} F(t+\xi) d t .
\end{aligned}
$$

Letting $B \rightarrow 0^{+}$, we see that $E_{1}=0$. Thus

$$
\limsup _{n \rightarrow \infty}\left|E_{\xi}\left(f, R_{n}\right)\right|=\lim _{n \rightarrow \infty}\left|E_{\xi}\left(f, R_{n}\right)\right|=0 . \quad \text { Q.E.D. }
$$

On combining Lemma 5 above, [1, Theorems 2 and 4] and the theorem in [2, p. $192]$, one easily proves the following result.

THEOREM 4. Suppose $(R)$ satisfies (3.1). For some fixed $\xi(0<\xi<T)$, let $f \in M_{d}(\xi)$. Then:

a. $\lim \inf _{n \rightarrow \infty}\left|R_{n}(f, \xi)-\int_{\xi}^{T} f(t) d t\right|=0$.

b. If $\xi / T$ is a rational (or respectively algebraic) number and if the abscissas $t_{i}$ used in the definition of rule $(R)$ are all rational (resp. algebraic) numbers, then

$$
\lim _{n \rightarrow \infty} R_{n}(f, \xi)=\int_{\xi}^{T} f(t) d t .
$$

This theorem can also be stated in a more general form which includes Theorem 4 above and Theorem 2 of Section 3.

THEOREM 5. Suppose $(R)$ satisfies (3.1). Suppose $f$ is an integrable function on $0 \leqq t \leqq T$ such that $f$ is continuous except at a finite set of singular points $\xi_{i}$ where $0 \leqq \xi_{1}<\xi_{2}<\cdots<\xi_{k} \leqq T$. Redefine $f(t)=0$ when $t=\xi_{i}$. If in each small onesided neighborhood of a singularity $\xi_{i}$ the function $|f(t)|$ can be dominated by a monotone integrable function, then:

a. $\lim \inf _{n \rightarrow \infty}\left|R_{n}(f)-\int_{0}^{T} f(t) d t\right|=0$.

b. If the numbers $\xi_{i} / T$ are all rational (or algebraic) and if the abscissas $t_{i}$ used in the rule $(R)$ are all rational (or respectively algebraic) numbers, then

$$
\lim _{n \rightarrow \infty} R_{n}(f)=\int_{0}^{T} f(t) d t .
$$

5. Numerical Examples. The data in [1] will be used to illustrate the theory given above. For the midpoint rule $M(f)=f\left(\frac{1}{2}\right)$ one has $H=h=T / n$. Since $-P_{n}(s)=s$ if $0<s<h / 2 ; s-h$ if $h / 2<s<h$, then $\left\|P_{n}\right\|=h / 2$ and $K=2$. Therefore, (3.7) has the form

$$
\left|E_{s}\left(f, M_{n}\right)\right| \leqq(h / 2) \int_{h}^{T}\left|f^{\prime}(t)\right| d t+\int_{0}^{h} F(t) d t+2 \int_{0}^{h / 2} F(t) d t .
$$

Table 1 contains data for the case

$$
\int_{0}^{1} t^{-1 / 2} d t=2
$$


TABLE 1

\begin{tabular}{cccccc}
\hline$n$ & $M_{n}\left(f_{0}\right)$ & Error & Th. Error & Ratio & Th. Ratio \\
\hline $2^{5}$ & 1.8931 & .1069 & .6763 & 1.414 & 1.414 \\
$2^{6}$ & 1.9244 & .0756 & .4815 & 1.413 & 1.414 \\
$2^{7}$ & 1.9465 & .0535 & .4321 & 1.415 & 1.414 \\
$2^{8}$ & 1.9622 & .0378 & .2427 & 1.416 & 1.414 \\
$2^{9}$ & 1.9733 & .0267 & .1720 & 1.413 & 1.414 \\
$2^{10}$ & 1.9811 & .0184 & .1218 & 1.410 & 1.414 \\
$2^{11}$ & 1.9866 & .0134 & .0864 & & \\
\hline
\end{tabular}

The fourth column is the theoretical error computed using (3.7). This error bound is seen to be pessimistic by a factor of 7 to 8 . Corollary 2 suggests that the error may be of the approximate form

$$
E_{s}\left(f, M_{n}\right)=C \sqrt{ } h \quad(h=T / n)
$$

for some constant $C>0$. The ratios $E_{0}\left(f, M_{n}\right) / E_{0}\left(f, M_{n+1}\right)$ are given in column five. The theoretical ratio computed using (5.2) is $\sqrt{ } 2$ (column six). It can be seen that (5.2) is approximately true with $C=.61$.

Table 2 contains similar data for (5.1) using the trapezoid rule (T), Simpson's rule $(S)$ and the Gaussian two-point rule $\left(G_{2}\right)$. The theoretical errors are good for the

TABLE 2

\begin{tabular}{cccccc}
\hline & Approx. & Error & Th. Error & Ratio & Th. Ratio \\
\hline $2^{5} \times T$ & 1.7418 & .2582 & .6031 & 1.414 & 1.414 \\
$2^{6} \times T$ & 1.8174 & .1826 & .4294 & 1.414 & 1.414 \\
$2^{7} \times T$ & 1.8709 & .1291 & .3055 & 1.414 & 1.414 \\
$2^{8} \times T$ & 1.9087 & .0913 & .2168 & 1.415 & 1.414 \\
$2^{9} \times T$ & 1.9355 & .0645 & .1537 & 1.414 & 1.414 \\
$2^{10} \times T$ & 1.9544 & .0456 & .1089 & & \\
$2^{5} \times S$ & 1.8427 & .1573 & 1.1792 & 1.413 & 1.414 \\
$2^{6} \times S$ & 1.8887 & .1113 & .8735 & 1.414 & 1.414 \\
$2^{7} \times S$ & 1.9213 & .0787 & .6198 & 1.415 & 1.414 \\
$2^{8} \times S$ & 1.9444 & .0556 & .4393 & 1.411 & 1.414 \\
$2^{9} \times S$ & 1.9606 & .0394 & .3113 & 1.412 & 1.414 \\
$2^{10} \times S$ & 1.9721 & .0297 & .2203 & & \\
$2 \times G_{2}$ & 1.7528 & .2472 & 7.6572 & 1.414 & 1.414 \\
$4 \times G_{2}$ & 1.8252 & .1748 & 5.4012 & 1.225 & 1.225 \\
$6 \times G_{2}$ & 1.8573 & .1427 & 4.4213 & 1.155 & 1.155 \\
$8 \times G_{2}$ & 1.8764 & .1236 & 3.8341 & 1.118 & 1.118 \\
$10 \times G_{2}$ & 1.8894 & .1106 & 3.4328 & 1.096 & 1.095 \\
$12 \times G_{2}$ & 1.8991 & .1009 & 3.1360 & 1.080 & 1.080 \\
$14 \times G_{2}$ & 1.9066 & .0934 & 2.9050 & 1.069 & 1.069 \\
$16 \times G_{2}$ & 1.9126 & .0874 & 1.7640 & & \\
\hline
\end{tabular}


trapezoid rule and progressively worse for Simpson and Gauss two-point. In all cases, (5.2) is approximately true, $E_{s}\left(f, T_{n}\right)=1.5 \sqrt{ } h, E_{s}\left(f, S_{n}\right)=.89 \sqrt{ } h$ and $E_{.}\left(f, n \times G_{2}\right)=.35 \sqrt{ } h$.

Data for the example

$$
\int_{0}^{t} t^{-1 / 2} \sin \left(t^{-1 / 2}\right) d t=1.008134
$$

are given in Table 3. For this case, our theory predicts convergence but yields no

TABLE 3

\begin{tabular}{lrrr}
\hline & Approx & \multicolumn{1}{c}{ Error } & Ratio \\
\hline $2^{5} \times S$ & 1.1234 & -.1153 & -1.195 \\
$2^{6} \times S$ & .9116 & .0965 & 2.726 \\
$2^{7} \times S$ & .9727 & .0354 & 1.053 \\
$2^{8} \times S$ & .9745 & .0336 & -2.800 \\
$2^{9} \times S$ & 1.0201 & -.0120 & 0.024 \\
$2^{10} \times S$ & 1.0586 & -.0507 & \\
$1 \times G_{48}$ & .9449 & .0632 & \\
$2 \times G_{48}$ & .9924 & .0157 & \\
$3 \times G_{48}$ & 1.0402 & -.0321 & \\
$4 \times G_{48}$ & .9301 & .0780 & \\
$5 \times G_{48}$ & 1.0597 & -.0516 & \\
$6 \times G_{48}$ & 1.0013 & .0068 & \\
$7 \times G_{48}$ & .9875 & .0206 & \\
$8 \times G_{48}$ & .9990 & .0091 & \\
$9 \times G_{48}$ & 1.0618 & -.0537 & \\
$10 \times G_{48}$ & 1.0211 & -.0130 & \\
\hline
\end{tabular}

useful information on convergence ratios. The data for Simpson's rules are taken from [1]. After an initial oscillation the rule $2^{n} \times S$ apparently settles down to slow but monotone convergence. The ratios indicate no pattern of convergence. Data for $n \times G_{48}$ are taken from [1] for $n=1,2,3,4$. The rest of the data was kindly supplied privately by Dr. P. Rabinowitz. In spite of the assurances of Theorem 2 , this quadrature oscillates without any real hint of convergence. Example 6 in $[1$, p. 383] with $A=-.5$ could be used to illustrate Theorem 4, part b. Again, the method converges (by Theorem 4 above) but convergence is much too slow to be practical.

Center for Dynamical Systems

Division of Applied Mathematics

Brown University

Providence, Rhode Island 02912

1. P. J. DAvis \& P. RabinowITZ, "Ignoring the singularity in approximate integration," J. Soc. Indust. Appl. Math. Ser. B Numer. Anal., v. 2, 1965, pp. 367-383. MR 33 \#3459.

2. P. RABINOWITZ, "Gaussian integration in the presence of a singularity," SIAM J. Numer. Anal., v. 4, 1967, pp. 191-201. MR 35 \#3881.

3. W. GAUTSCHI, "Numerical quadrature in the presence of a singularity," SIAM J. Numer. Anal., v. 4, 1967, pp. 357-362. MR 36 \#1103.

4. A. SARD, Linear Approximation, Math. Surveys, no. 9, Amer. Math. Soc., Providence, R.I., 1963. MR 28 \#1429. 